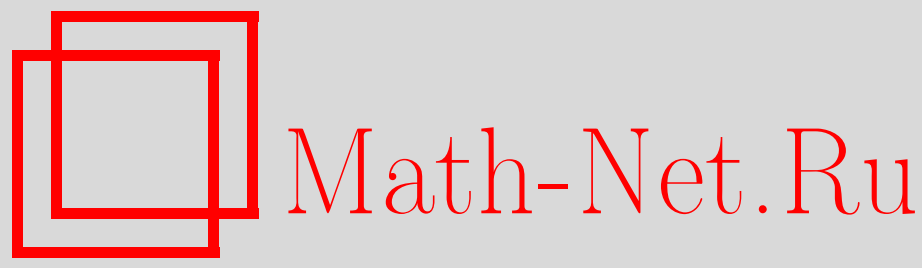

Е. В. Трифонов, Семейства точных решений для линейных и нелинейных волновых уравнений с переменной скоростью звука и их использование для решения начально-краевых задач, ТМФ, 2017, том 192, номер 1, 41-50

DOI: https://doi.org/10.4213/tmf9263

Использование Общероссийского математического портала Math-Net.Ru подразумевает, что вы прочитали и согласны с пользовательским соглашением http://www . mathnet.ru/rus/agreement

Параметры загрузки:

IP: 35.173 .219 .149

26 апреля 2023 г., 14:13:25

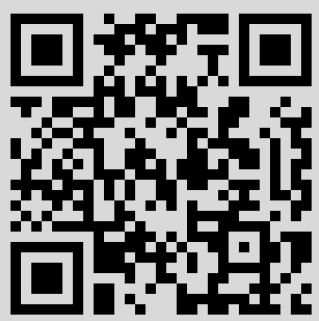




\title{
СЕМЕЙСТВА ТОЧНЫХ РЕШЕНИЙ ДЛЯ ЛИНЕЙНЫХ И НЕЛИНЕЙНЫХ ВОЛНОВЫХ УРАВНЕНИЙ \\ С ПЕРЕМЕННОЙ СКОРОСТЬЮ ЗВУКА И ИХ ИСПОЛЬЗОВАНИЕ ДЛЯ РЕШЕНИЯ НАЧАЛЬНО-КРАЕВЫХ ЗАДАЧ
}

\begin{abstract}
Предложена процедура размножения решений линейных и нелинейных одномерных волновых уравнений. При этом скорость звука может быть произвольной функцией от одной переменной. Получены точные решения. Показано, что функциональный ряд из этих решений можно использовать для решения начально-краевых задач. Для этого введено специальное скалярное произведение.
\end{abstract}

Ключевые слова: точные решения, волновое уравнение, преобразования Беклунда.

DOI: https://doi.org/10.4213/tmf9263

\section{1. ВВЕДЕНИЕ}

В данной работе рассматриваются линейные и нелинейные пространственно-одномерные волновые уравнения, описывающие акустические волны. Для них выводятся автопреобразования Беклунда и с их помощью получаются семейства точных решений, которые затем используются для аппроксимации начально-краевых задач. Заметим, что отдельные функции из семейств, вообще говоря, не удовлетворяют граничным условиям, но из них составляется функциональный ряд таким образом, чтобы эти условия выполнялись с достаточной точностью.

Разные варианты преобразований Беклунда ранее применялись в физике сплошных сред. В посвященных этой тематике работах нелинейные уравнения линеаризовались с помощью преобразования годографа. Для линейной системы с переменными коэффициентами выводились условия на коэффициенты в случае, когда существуют преобразования Беклунда к уравнениям канонического вида. Подробный

* Институт автоматики и процессов управления ДВО РАН, Владивосток, Россия. E-mail: ev.trifonov@gmail.com

† Дальневосточный федеральный университет, Владивосток, Россия 
обзор данного подхода можно найти в монографии [1]. Заметим, что упомянутые условия приводят к ограничениям на вид уравнения состояния и, соответственно, скорости звука.

Отличие преобразований Беклунда, полученных в настоящей работе, состоит в том, что во-первых, они являются автопреобразованиями, а во вторых - справедливы для любой функциональной зависимости скорости звука.

\section{2. ОСНОВНЫЕ УРАВНЕНИЯ}

Поскольку вид нелинейных волновых уравнений акустики не является единственным, остановимся на их выводе. Кроме того, системы первого порядка, полученные при выводе, используются в дальнейшем при нахождении точных решений.

Динамику среды в звуковой волне можно считать адиабатической, а следовательно, баротропной. Рассмотрим одномерное движение баротропного невязкого газа, описываемое уравнениями

$$
\begin{aligned}
\frac{\partial \rho}{\partial t}+\frac{\partial(\rho u)}{\partial x} & =0 \\
\frac{\partial(\rho u)}{\partial t}+\frac{\partial\left(\rho u^{2}+p\right)}{\partial x} & =0 .
\end{aligned}
$$

Если предположить, как это принято в акустике, что смещения частиц среды малы по сравнению с длиной волны, то конвективным слагаемым можно пренебречь [2]. Обозначим $\left.\frac{\partial p}{\partial \rho}\right|_{S}=c^{2}(\rho)$. Тогда уравнения принимают вид

$$
\begin{aligned}
\frac{\partial \rho}{\partial t}+\frac{\partial \mu}{\partial x} & =0, \\
\frac{\partial \mu}{\partial t}+c^{2}(\rho) \frac{\partial \rho}{\partial x} & =0,
\end{aligned}
$$

где $\mu=\rho u-$ плотность импульса, или

$$
\begin{aligned}
\frac{1}{C^{2}(p)} \frac{\partial p}{\partial t}+\frac{\partial \mu}{\partial x} & =0 \\
\frac{\partial \mu}{\partial t}+\frac{\partial p}{\partial x} & =0
\end{aligned}
$$

Функции $C$ и $c$ представляют собой одну и ту же скорость звука, но выраженную в различных переменных: $C(p)$ зависит от давления $p$, а $c(\rho)$ зависит от плотности $\rho$. Справедливо тождество $C(p(\rho))=c(\rho)$. Например, для идеального газа

$$
p \sim \rho^{\gamma}, \quad c(\rho) \sim \rho^{(\gamma-1) / 2}, \quad C(p) \sim p^{(\gamma-1) / 2 \gamma},
$$

где $\gamma$ - показатель адиабаты.

Из равенства смешанных частных производных функции $\mu$ получаем нелинейные волновые уравнения для плотности

$$
\rho_{t t}-\left(c^{2}(\rho) \rho_{x}\right)_{x}=0
$$

и давления

$$
\left(\frac{p_{t}}{C^{2}(p)}\right)_{t}-p_{x x}=0
$$


Эти уравнения возникают также при описании распространения электромагнитных волн в средах с нелинейной поляризацией и проводимостью [3].

Из физических соображений можно предположить вид преобразования между нелинейными волновыми уравнениями (7) и (8):

$$
\rho=\int \frac{d p}{C^{2}(p)}, \quad p=\int c^{2}(\rho) d \rho .
$$

Это утверждение легко проверить непосредственно.

Обычно в акустике также используют предположение о слабой интенсивности волны, что приводит к линейным уравнениям. Однако в данной работе мы рассматриваем и линейные, и нелинейные волновые уравнения.

Скорость звука может меняться в пространстве, в таком случае волны описываются линейной системой с переменными коэффициентами

$$
\begin{aligned}
\frac{1}{C^{2}(x)} \frac{\partial p}{\partial t}+\frac{\partial \mu}{\partial x} & =0 \\
\frac{\partial \mu}{\partial t}+\frac{\partial p}{\partial x} & =0
\end{aligned}
$$

если скорость звука меняется во времени, тогда применима линейная система

$$
\begin{aligned}
\frac{\partial \rho}{\partial t}+\frac{\partial \mu}{\partial x} & =0 \\
\frac{\partial \mu}{\partial t}+c^{2}(t) \frac{\partial \rho}{\partial x} & =0 .
\end{aligned}
$$

\section{3. АВТОПРЕОБРАЗОВАНИЯ БЕКЛУНДА ДЛЯ ЛИНЕЙНЫХ СИСТЕМ И СЕМЕЙСТВА ТОЧНЫХ РЕШЕНИЙ}

С системой $(10),(11)$ можно связать дифференциальные формы $d p-p_{x} d x-p_{t} d t$ и $d \mu-\mu_{x} d x-\mu_{t} d t$. Из исходной системы мы можем найти соотношения между производными, с учетом этого факта получаем равенства

$$
\begin{aligned}
d p & =a d t-b d x \\
d \mu & =b d t-\frac{a}{C^{2}(x)} d x
\end{aligned}
$$

где $a$ и $b$ - некоторые неизвестные функции. Если это семейство дифференциальных форм является вполне интегрируемым, то мы можем вывести из нее искомые функции $p$ и $\mu$. Условия интегрируемости имеют вид

$$
\begin{aligned}
\frac{\partial b}{\partial t}+\frac{\partial a}{\partial x} & =0 \\
\frac{\partial a}{\partial t}+C^{2}(x) \frac{\partial b}{\partial x} & =0 .
\end{aligned}
$$


Таким образом, уравнения совместности $(16),(17)$ для функций $a$ и $b$ имеют тот же вид, что и система $(10),(11)$. Следовательно, если у нас есть решение $\left(p_{0}, \mu_{0}\right)$, то мы можем выбрать $a=p_{0}, b=\mu_{0}$; тогда система (14), (15) оказывается совместной и из нее можно найти $(p, \mu)$ - новое решение системы $(10),(11)$. Поскольку система (16), (17) была получена в качестве условий совместности и совпала с исходной системой $(10),(11)$, изложенную процедуру справедливо считать автопреобразованием Беклунда [4].

Возьмем в качестве начального решения константы. Для интегрирования дифференциальных форм используем следующее легко доказываемое утверждение: если дифференциальная форма $d u=f(x, t) d x+g(x, t) d t$ вполне интегрируема, то

$$
u=\int_{0}^{x} f(x, 0) d x+\int_{0}^{t} g(x, t) d t .
$$

В этом случае мы применяем интегрирование по пути, имеющему форму в виде "уголка". В результате получаем семейство решений

$$
\begin{gathered}
p=k_{1}, \quad \mu=k_{2}, \\
p=k_{1} t-k_{2} x+k_{3}, \quad \mu=k_{2} t-k_{1} \int \frac{d x}{C^{2}(x)}+k_{4}, \\
p=k_{1} \int d x \int \frac{d x}{C^{2}(x)}-k_{4} x+k_{1} \frac{t^{2}}{2}-k_{2} x t+k_{3} t+k_{5}, \\
\mu=k_{2} \int \frac{x d x}{C^{2}(x)}-k_{3} \int \frac{d x}{C^{2}(x)}+k_{2} \frac{t^{2}}{2}-k_{1} t \int \frac{d x}{C^{2}(x)}+k_{4} t+k_{6},
\end{gathered}
$$

где $k_{i}=$ const. Этот процесс можно продолжать и дальше, получая новые точные решения. При этом будет увеличиваться количество произвольных параметров.

Аналогично можно поступить с системой (12), (13). С ней ассоциируются дифференциальные формы $d \rho-\rho_{x} d x-\rho_{t} d t$ и $d \mu-\mu_{x} d x-\mu_{t} d t$. С учетом исходной системы записываем уравнения

$$
\begin{aligned}
& d \rho=a d x-b d t \\
& d \mu=b d x-c^{2}(t) a d t
\end{aligned}
$$

где $a$ и $b$ - некоторые неизвестные функции. Условия интегрируемости имеют вид

$$
\begin{aligned}
\frac{\partial a}{\partial t}+\frac{\partial b}{\partial x} & =0 \\
\frac{\partial b}{\partial t}+c^{2}(t) \frac{\partial a}{\partial x} & =0 .
\end{aligned}
$$

Таким образом, уравнения совместности (20), (21) для функций $a$ и $b$ записываются так же, как и система $(12),(13)$. Следовательно, если у нас есть решение $\left(\rho_{0}, \mu_{0}\right)$, то, полагая $a=\rho_{0}, b=\mu_{0}$, мы найдем из системы $(18),(19)(\rho, \mu)$ - новое решение системы (12), (13). 
В качестве "затравочного" решения можно взять константы. Тогда последовательно получаем решения

$$
\begin{gathered}
\rho=k_{1}, \quad \mu=k_{2}, \\
\rho=k_{1} x-k_{2} t+k_{3}, \quad \mu=k_{2} x-k_{1} \int c^{2}(t) d t+k_{4}, \\
\rho=k_{1} \frac{x^{2}}{2}-k_{2} x t+k_{1} \int\left(\int c^{2}(t) d t\right) d t+k_{3} x-k_{4} t+k_{5}, \\
\mu=k_{2} \frac{x^{2}}{2}-k_{1} x \int c^{2}(t) d t+k_{2} \int t c^{2}(t) d t+k_{4} x-k_{3} \int c^{2}(t) d t+k_{6} .
\end{gathered}
$$

\section{4. ТОЧНЫЕ РЕШЕНИЯ}

\section{ДЛЯ НЕЛИНЕЙНЫХ ВОЛНОВЫХ УРАВНЕНИЙ}

Решения нелинейных уравнений (3), (4) можно интерпретировать как интегральное многообразие системы дифференциальных форм

$$
\begin{aligned}
d \rho \wedge d x+d t \wedge d \mu & =0, \\
d \mu \wedge d x+c^{2}(\rho) d t \wedge d \rho & =0,
\end{aligned}
$$

проецирующееся на плоскость $x, y$. Если рассматривать $x$ и $y$ как функции от $\rho$ и $\mu$, мы получаем линейную систему уравнений, эквивалентную исходной:

$$
\begin{aligned}
x_{\mu}+t_{\rho} & =0, \\
x_{\rho}+c^{2}(\rho) t_{\mu} & =0 .
\end{aligned}
$$

Эта система есть не что иное, как уравнения (12), (13) в других обозначениях: системы переходят друг в друга при преобразовании

$$
\rho \mapsto t, \quad t \mapsto \rho, \quad x \mapsto \mu, \quad \mu \mapsto x .
$$

По существу, выше приведено альтернативное изложение метода годографа [5] с использованием дифференциальных форм.

Аналогично, нелинейные уравнения (5), (6) преобразуются в линейную систему (10), (11) при замене

$$
p \mapsto x, \quad x \mapsto p, \quad t \mapsto \mu, \quad \mu \mapsto t .
$$

Таким образом, полученные ранее решения линейных волновых уравнений можно использовать для нахождения решений нелинейных волновых уравнений. Например, выражения

$$
\begin{aligned}
& t=k_{1} \frac{\mu^{2}}{2}-k_{2} \mu \rho+k_{1} \int\left(\int c^{2}(\rho) d \rho\right) d \rho+k_{3} \mu-k_{4} \rho+k_{5}, \\
& x=k_{2} \frac{\mu^{2}}{2}-k_{1} \mu \int c^{2}(\rho) d \rho+k_{2} \int \rho c^{2}(\rho) d \rho+k_{4} \mu-k_{3} \int c^{2}(\rho) d \rho+k_{6}
\end{aligned}
$$


представляют в неявном виде решения уравнений (3), (4), а выражения

$$
\begin{aligned}
& x=k_{1} \int d p \int \frac{d p}{C^{2}(p)}-k_{4} p+k_{1} \frac{\mu^{2}}{2}-k_{2} p \mu+k_{3} \mu+k_{5} \\
& t=k_{2} \int \frac{p d p}{C^{2}(p)}-k_{3} \int \frac{d p}{C^{2}(p)}+k_{2} \frac{\mu^{2}}{2}-k_{1} \mu \int \frac{d p}{C^{2}(p)}+k_{4} \mu+k_{6}
\end{aligned}
$$

дают решения уравнений (5), (6).

\section{5. ИСПОЛЬЗОВАНИЕ ДЛЯ ПОЛУЧЕНИЯ РЕШЕНИЯ НАЧАЛЬНО-КРАЕВЫХ ЗАДАЧ}

Рассмотрим волновое уравнение с переменной скоростью звука, вытекающее из системы (10), (11):

$$
p_{t t}-c^{2}(x) p_{x x}=0
$$

В уравнении (24) переменные разделяются. Однако соответствующую задачу Штурма-Лиувилля в общем случае нельзя решить в явном виде. С другой стороны, полученные нами семейства точных решений выражаются через квадратуры, но они обладают и существенным недостатком - не удовлетворяют типичным граничным условиям (например, для струны с закрепленными концами).

Преодолеть этот недостаток предлагается следующим образом. По существу, частичные суммы обобщенного ряда Фурье, возникающие при решении начально-краевых задач, точно удовлетворяют граничным условиям и приближенно - начальным условиям. Мы строим из нашего семейства ряд по ортонормированным функциям, который приближенно удовлетворяет и начальным, и граничным условиям. Погрешность будет убывать при возрастании числа членов ряда.

Ограничим время, в течение которого справедливы полученные решения, некоторой постоянной $T$. Мы можем положить без ущерба для общности рассуждений, что $T=1$, проведя, если необходимо, замену переменной времени $t$. Рассмотрим следующую начально-краевую задачу для уравнения (24):

$$
\begin{aligned}
& p(x, 0)=p_{0}(x), \quad p_{t}(x, 0)=p_{1}(x), \quad x \in[\alpha, \beta], \\
& p(\alpha, t)=l(t), \quad p(\beta, t)=r(t), \quad t \in[0,1] .
\end{aligned}
$$

Например, для струны с закрепленными концами $l(t)=r(t)=0$. Пусть $u(x, t)-$ функция, удовлетворяющая уравнению (24) точно, а условиям (25), (26) - приближенно.

В качестве меры погрешности используем функционал

$$
\begin{aligned}
\int_{\alpha}^{\beta}\left(u(x, 0)-p_{0}(x)\right)^{2} d x & +\int_{\alpha}^{\beta}\left(u_{t}(x, 0)-p_{1}(x)\right)^{2} d x+ \\
& +\int_{0}^{1}(u(\alpha, t)-l(t))^{2} d t+\int_{0}^{1}(u(\beta, t)-r(t))^{2} d t .
\end{aligned}
$$


С этим функционалом можно ассоциировать скалярное произведение

$$
\begin{aligned}
\langle v, w\rangle= & \int_{\alpha}^{\beta} v(x, 0) w(x, 0) d x+\int_{\alpha}^{\beta} v_{t}(x, 0) w_{t}(x, 0) d x+ \\
& +\int_{0}^{1} v(\alpha, t) w(\alpha, t) d t+\int_{0}^{1} v(\beta, t) w(\beta, t) d t .
\end{aligned}
$$

С помощью него минимизацию функционала погрешности можно сформулировать в виде

$$
\left\langle u-u_{0}, u-u_{0}\right\rangle \rightarrow \min .
$$

Пусть $f_{1}, f_{2}, \ldots, f_{n}$ - ортонормированная система функций по отношению к этому скалярному произведению. Тогда ряд $\sum_{i=1}^{m} c_{i} f_{i}$ с коэффициентами $c_{i}$, определенными как $c_{i}=\left\langle u_{0}, f_{i}\right\rangle$, минимизирует функционал погрешности.

Для иллюстрации предложенного подхода рассмотрим такой частный случай волнового уравнения (24) с непостоянной скоростью звука, что соответствующую задачу Штурма-Лиувилля все-таки можно решить аналитически. Положим скорость звука равной $c(x)=x$, тогда можно найти точное решение

$$
p(x, t)=\sqrt{x} \cos \left(t \sqrt{\frac{1}{4}+\frac{\pi^{2}}{\log ^{2} 2}}\right) \sin \left(\pi \log _{2} x\right) .
$$

Оно удовлетворяет начальным и граничным условиям

$$
p_{0}=\sqrt{x} \sin \left(\pi \log _{2} x\right), \quad p_{1}=0, \quad l(t)=r(t)=0, \quad \alpha=1, \quad \beta=2 .
$$

Решим теперь это уравнение с условиями (30) в виде ряда по функциям из семейства построенных нами решений и сравним с точным решением (29). Прежде всего построим семейство решений для скорости звука $c(x)=x$ по процедуре, описанной в предыдущих разделах, полагая константы удовлетворяющими условиям

$$
k_{1}=k_{2}=1, \quad k_{i}=0, \quad i=3,4, \ldots .
$$

Получаем, что

$$
u_{1}=1, \quad u_{2}=t-x, \quad u_{3}=\frac{t^{2}}{2}-t x-\log x, \quad \ldots .
$$

Далее, строим на этом семействе ортонормированное относительно скалярного произведения (28) множество решений. В нашем случае $\alpha=1, \beta=2$. Введем новое семейство функций по формулам

$$
F_{i}=u_{i}-\sum_{j=1}^{i-1} d_{j} u_{j}
$$

Константы $d_{i}$ определим из соотношений

$$
\left\langle F_{i}, u_{j}\right\rangle=0, \quad j=1,2, \ldots, i-1 .
$$

Далее, строим нормированное семейство

$$
f_{i}=\frac{F_{i}}{\sqrt{\left\langle F_{i}, F_{i}\right\rangle}} .
$$




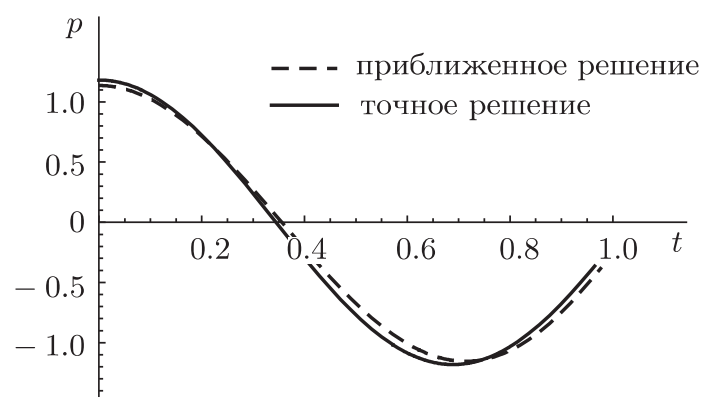

Рис. 1. Точное и приближенное решения в середине отрезка.

Таким образом, находим, что

$$
f_{1}=\frac{1}{\sqrt{3}}, \quad f_{2}=\frac{6 t-6 x+7}{\sqrt{69}}, \quad \ldots .
$$

Наконец, записываем представление для решения в виде

$$
p=\sum_{i=1}^{m}\left\langle u_{0}, f_{i}\right\rangle f_{i}
$$

где скалярное произведение дается выражением (28).

Динамика волны в середине отрезка хорошо приближается уже при достаточно небольшой длине ряда, как показано на рис. 1 для $m=11$. При $m \geqslant 15$ графики визуально не различаются. Вопросы сходимости и свойств этого ряда могут являться предметом дальнейших исследований.

\section{6. ЗАДАЧА КОШИ \\ ДЛЯ НЕЛИНЕЙНОГО ВОЛНОВОГО УРАВНЕНИЯ}

Рассмотрим нелинейное волновое уравнение (8) с начальными условиями

$$
p(x, 0)=p_{0}(x), \quad p_{t}(x, 0)=p_{1}(x) .
$$

Из начального условия для $p_{t}$ можно получить условие $\mu(x, 0)=\mu_{0}(x)$ для нелинейной системы (5), (6).

Ранее мы получили семейства точных решений этого уравнения, пример приведен в (23). В общем случае выражения для $x=X(p, \mu, \mathbf{k}), t=T(p, \mu, \mathbf{k})$ линейно зависят от произвольных констант $k_{i}$. Одна из трудностей нелинейного случая связана с тем, что решения представляются в неявном виде. Но на конечном этапе эти зависимости необходимо обратить, выразив $p$ через $x$ и $t$, что при произвольных константах сделать затруднительно. Поэтому представляется целесообразным учесть начальные условия для неявного вида решений, и только потом обратить зависимости. 


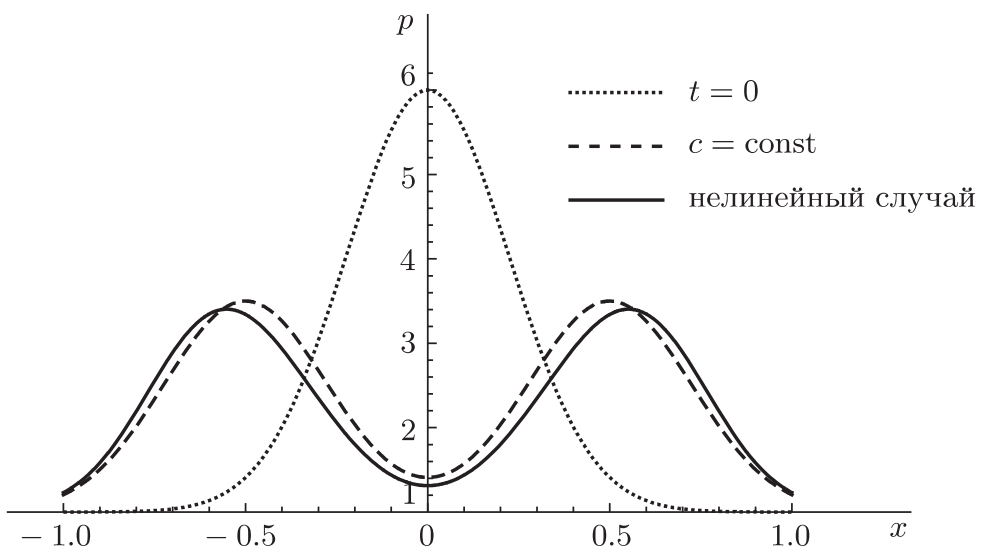

Рис. 2. Эволюция волновых импульсов.

Достичь этой цели, можно, например, с помощью минимизации функционала

$$
\int_{\alpha_{j}}^{\beta_{j}}\left[\left(X\left(p_{0}(x), \mu_{0}(x), \mathbf{k}\right)-x\right)^{2}+T^{2}\left(p_{0}(x), \mu_{0}(x), \mathbf{k}\right)\right] d x \rightarrow \min _{\mathbf{k}}
$$

где $\alpha_{j}$ и $\beta_{j}$ - границы интервалов монотонности поведения функций. В самом деле, для немонотонных функций одному уровню давления соответствуют несколько значений координаты $x$, следовательно, понадобится и несколько зависимостей.

В результате минимизации возникает линейная алгебраическая система на константы $k_{i}$. Если решения представить в виде линейной комбинации ортонормированных функций, то матрица этой системы станет единичной.

Заметим, что данный подход легко модифицировать, чтобы учесть и граничные условия. Для этого надо добавить соответствующие члены в целевую функцию минимизации аналогично тому, как это было сделано в предыдущем разделе.

В качестве примера приведем расчет динамики гауссовского начального распределения в воздушной среде с помощью указанной выше процедуры. Единицы измерения выбраны таким образом, что $p_{\infty}=1, C(p)=p^{(\gamma-1) / 2 \gamma}$. Здесь $\gamma-$ показатель адиабаты для воздуха, $\gamma=1.4$.

На рис. 2 представлены распределения давления в моменты $t=0$ и $t=1 / 2$, причем для сравнения представлен и случай постоянной скорости звука $c=1=$ const. Влияние нелинейности выражается в более быстром движении пиков и укручении передних фронтов.

\section{7. ЗАКЛЮЧЕНИЕ}

В работе были получены новые классы точных решений волновых уравнений с непостоянной скоростью звука, в том числе нелинейных. Произвольные константы, входящие в эти решения, можно выбрать таким образом, чтобы получать решения начально-краевых задач. 


\section{Список литературы}

[1] C. Rogers, W. F. Shadwick, Bäcklund Transformations and Their Applications, Mathematics in Science and Engineering, 161, Academic Press, New York, 1982.

[2] М. А. Исакович, Общая акустика, Наука, М., 1973.

[3] В. М. Журавлев, "Многомерные нелинейные волновые уравнения с многозначными решениями", ТМФ, 174:2 (2013), 272-284.

[4] G.L. Lamb, Jr., "Bäcklund Transforms at the Turn of the Century", Bäcklund Transformations, the Inverse Scattering Method, Solitons, and Their Applications, Lecture Notes in Mathematics, 515, ed. R. M. Miura, Springer, Berlin, 1976, 69-79.

[5] Б. Л. Рождественский, Н. Н. Яненко, Системы квазилинейных уравнений и их приложения к газовой динамике, Наука, М., 1978.

Поступила в редакцию 21.07.2016, после доработки 16.11.2016 